\title{
Banding the Right Ventricular Assist Device Outflow Conduit: is it really necessary with current devices?
}

${ }^{1}$ Casey Lo MBBS; ${ }^{2,3}$ Shaun Gregory PhD; ${ }^{2}$ Michael Stevens BEng; ${ }^{4}$ Deirdre Murphy FCICM; ${ }^{1}$ Silvana Marasco FRACS

${ }^{1}$ Sir Charles James Officer Brown Department of Cardiothoracic Surgery, The Alfred Hospital, Prahran, Victoria, Australia ${ }^{3}$ Innovative Cardiovascular Engineering and Technology Laboratory, Critical Care Research Group, The Prince Charles Hospital, Brisbane, Queensland, Australia

${ }^{3}$ School of Medicine, The University of Queensland, Brisbane, Queensland, Australia

${ }^{4}$ Cardiothoracic Intensive Care Unit, The Alfred Hospital, Prahran, Victoria, Australia

\section{$\underline{\text { Abstract }}$}

Implantable left ventricular assist devices (LVADs) have been adapted clinically for right-sided mechanical circulatory support (RVAD). Previous studies on RVAD support have established the benefits of outflow cannula restriction and rotational speed reduction, and recent literature has focused on assessing either the degree of outflow cannula restriction required to simulate left-sided afterload, or the limitation of RVAD rotational speeds. Anecdotally, the utility of outflow cannula restriction has been questioned, with suggestion that banding may be unnecessary and may be replaced simply by varying the outflow conduit length. Furthermore, many patients have a high pulmonary vascular resistance (PVR) at the time of VAD insertion that reduces with pulmonary vascular bed remodelling. It is therefore important to assess the potential changes in flow through a RVAD as PVR changes. In this in-vitro study, we observed the use of dual HeartWare HVAD devices (HeartWare Inc., Framingham MA, USA) in BiVAD configuration. We assessed the pumps' ability to maintain haemodynamic stability with and without banding; and with varying outflow cannulae length $(20,40$ and $60 \mathrm{~cm})$. Increased length of the outflow conduit was found to produce significantly increased afterload to the device, but this was not found to be necessary to maintain the device within the manufacturers recommended operational parameters under a simulated normal physiological setting of mild and severe RV failure. We hypothesise that $40 \mathrm{~cm}$ of outflow conduit, laid down along the diaphragm and then up over the RV to reach the pulmonary trunk, will generate sufficient resistance to maintain normal pump function. 


\section{Introduction}

Ventricular assist devices (VADs) implanted in the left ventricle (LVAD) have been demonstrated to provide both statistically and clinically significant improvement in the life expectancy and quality of life for end-stage heart failure patients (1). However, with the high incidence of subsequent right ventricular failure and the associated increased morbidity and mortality (2), and evidence of improved outcomes with early biventricular mechanical support $(3,4)$, the ongoing development of mechanical biventricular assistance techniques remains pertinent.

All current commercially available continuous-flow centrifugal VADs for longterm implantation are designed for left-sided use $(5,6)$. As such, their design specifications are tailored to a pump against left-sided afterload. For the purpose of right ventricular support, implantable VADs are thus adapted to function in the setting of the lower resistance of the right-side. This can either be achieved by decreasing pump speed or modification of the outflow conduit to avoid overpumping.

Decreasing RVAD pump speed to below manufacturer's specifications moves away from the design point for pump function, and can influence pump washout and thus thrombus formation within the pump (7). Furthermore, with hydrodynamic suspension of the impeller within the device, insufficient pump speed may lead to impeller instability (7). Previous in-vitro studies on biventricular support using dual LVADs had already established the benefits of outflow cannula restriction and rotational speed reduction. Recent literature has thus focused on assessing either the degree of outflow cannula restriction required to simulate left-sided afterload, or the limitation of dual VAD rotational speeds (5). However, anecdotally, the utility of outflow cannula restriction has been questioned, with suggestion that banding may be entirely unnecessary in an in-vivo setting. Although not described in the literature, there have been anecdotal suggestions that, by Poiseuille's Law, the same outflow resistance may 
be achieved by increasing graft length. Furthermore, many patients have a high pulmonary vascular resistance (PVR) at the time of VAD insertion that reduces over time. It is, therefore, important to assess the potential changes in flow through a RVAD as PVR changes, and how different outflow conduits may affect this.

In this in-vitro study, we observed the use of dual HeartWare HVAD devices (HeartWare Inc., Framingham MA, USA) in BiVAD configuration in a mockcirculation loop (MCL). The manufacturer's specified operating speed for these devices are 2400 to 3200 RPM. We assessed the pumps' ability to maintain haemodynamic stability with and without banding; and with varying outflow cannulae length. We aimed to assess the suitability of the HeartWare HVAD for use in dual device biventricular support and establish the optimal settings for such use with various levels of PVR. We hypothesise that graft length will have a demonstrable impact on outflow conduit resistance, but that it would not equal that of banding. This study will demonstrate whether the reduced incidence will still produce conditions that allow for normal pump function. 


\section{$\underline{\text { Methods }}$}

\section{Mock Circulation Loop}

A physical five-element Windkessel mock circulation loop (MCL) including systemic and pulmonary circulations was used for this study (Figure 1) $(8,9)$. In brief, ventricular systole was controlled through a series of electropneumatic regulators (ITV2030-012BS5, SMC Pneumatics, Tokyo, Japan) and 3/2 way solenoid valves (VT325-035DLS, SMC Pneumatics, Tokyo, Japan) to provide passively filled heart chambers and variable contractility, heart rate and systolic time. Heart rate and systolic time were maintained at 60 beats per minute and $35 \%$ respectively throughout this study. A Starling response was implemented in both left and right ventricles, which actively controlled ventricular contractility (through electropneumatic regulator supply current) based on ventricular preload (10). Mechanical check valves were used to simulate the mitral, aortic, tricuspid and pulmonary valves to ensure unidirectional flow throughout the circuit. Four independent Windkessel chambers were employed to simulate lumped systemic and pulmonary arterial and venous compliance. Socket valves (VMP025.03X.71, Alb. Klein Ohio, Plain City, OH) allowed easy manipulation of systemic and pulmonary vascular resistance respectively. The working fluid throughout this study was a water/glycerol mixture $(60 / 40 \%$ by mass) with similar viscosity and density to that of blood $(11,12)$. Inflow and outflow cannulation for the LVAD and RVAD was achieved via the ventricles for inflow and aorta / pulmonary artery for outflow. 


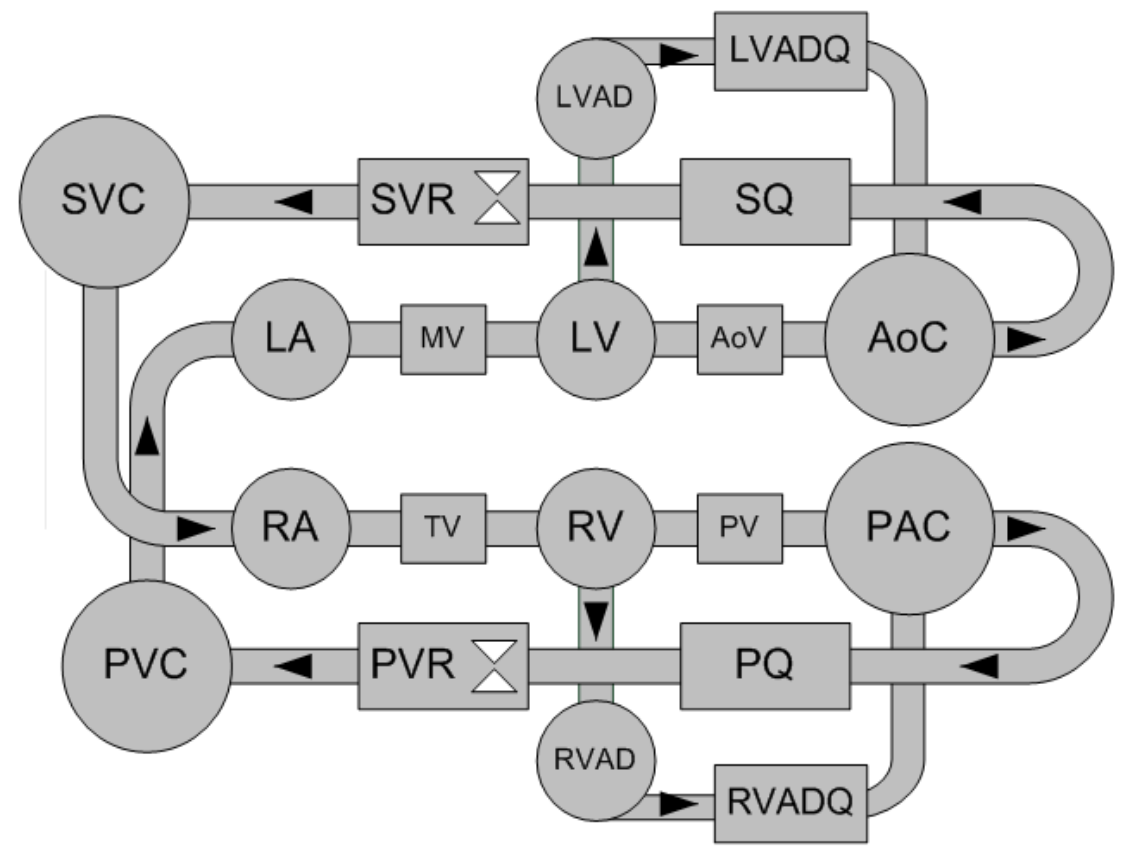

Figure 1: Schematic of the MCL setup for evaluation of a dual HeartWare biventricular support system. LA - left atrium, MV - mitral valve, LV - left ventricle, AoV - aortic valve, AoC - aortic compliance chamber, SQ - systemic flow meter, SVR - systemic vascular resistance valve, SVC systemic venous compliance chamber, $R A$ - right atrium, TV - tricuspid valve, $R V$ - right ventricle, PV - pulmonary valve, PAC - pulmonary arterial compliance chamber, PQ - pulmonary flow meter, PVR pulmonary vascular resistance valve, PVC - pulmonary venous compliance chamber, LVAD - left ventricular assist device, LVADQ - left ventricular assist device flow meter, RVAD - right ventricular assist device, RVADQ - right ventricular assist device flow meter.

\section{Dual LVAD Evaluation}

Severe LV failure was simulated in the MCL and supported using a HeartWare HVAD device at 2920 RPM to maintain $5 \mathrm{~L} / \mathrm{min}$ aortic flow and mean aortic pressure (MAP) of $80 \mathrm{mmHg}$. Haemodynamics for supported and unsupported conditions are highlighted in Table 1. Severe RV failure was simulated and supported using a second HeartWare HVAD device, under test conditions of: unrestricted $10 \mathrm{~mm}$ diameter outflow cannulae of 20, 40 and $60 \mathrm{~cm}$ lengths and a $20 \mathrm{~cm}$ length cannula restricted to $5 \mathrm{~mm}$ diameter. The shorter lengths were selected for clinical feasibility, while the long length was tested as an academic exercise to trend the effect of various graft lengths on pump function. Outflow cannulae were standard Dacron $®$ (Kennesaw, Georgia, USA) vascular grafts coated in petroleum jelly and wrapped loosely with cling-wrap for waterproofing. The conduits were carefully placed to avoid kinking. 


\begin{tabular}{cccccccccccc}
\hline Condition & MAP & MPAP & MSQ & MPQ & LAP & RAP & HR & SVR & PVR & LVADS & RVADS \\
& $m m H g$ & $m m H g$ & $L / m i n$ & $L / m i n$ & $m m H g$ & $m m H g$ & $B P M$ & dyne.s.cm ${ }^{-5}$ & dyne.s.cm & rpm & rpm \\
\hline MRHF & 56 & 13 & 2.8 & 2.8 & 9.5 & 8.9 & 60 & 1350 & 100 & - & - \\
MRHF-S & 80 & 16 & 5.0 & 5.0 & 10.8 & 9.3 & 60 & 1350 & 80 & 2920 & 2420 \\
SRHF & 48 & 9 & 2.3 & 2.3 & 6 & 9.6 & 60 & 1350 & 100 & - & - \\
SRHF-S & 80 & 16 & 5.0 & 5.0 & 10.7 & 8.5 & 60 & 1350 & 80 & 2920 & 2200 \\
\hline
\end{tabular}

Table 1 - Haemodynamic parameters for steady state conditions of mild (MRHF) and severe (SRHF) right heart failure models with (MRHF-S and SRHF-S respectively) and without dual rotary blood pump support. Note severe left heart failure was simulated in all conditions, and $20 \mathrm{~cm}$ unbanded RVAD outflow conduit was used. MAP - mean aortic pressure, MPAP - mean pulmonary artery pressure, MSQ - mean systemic flow rate, MPQ - mean pulmonary flow rate, HR - heart rate, SVR systemic vascular resistance, PVR - pulmonary vascular resistance, LVADS - LVAD rotational speed, RVADS - RVAD rotational speed, rpm - revolutions per minute.

For each cannula length and banding simulation, PVR was successively increased from 40 to 560 Dynes.s.cm ${ }^{-5}$ (0.5 to 7 Wood units) in 40 Dynes.s.cm ${ }^{-5}$ (0.5 Wood unit) intervals. RVAD speed was manually manipulated to maintain haemodynamic stability with balanced systemic and pulmonary flow rates of 5.0 L/min for each level of PVR. All other conditions were kept consistent between tests. The experiment was repeated in mild RV systolic dysfunction to assess the clinical applicability of the findings.

\section{Data Acquisition}

Haemodynamic and VAD parameters were captured at $100 \mathrm{~Hz}$ using a dSPACE acquisition system (DS1104, dSPACE, Wixom, MI, USA). Systemic and pulmonary flow rates were recorded using magnetic flow meters (IFC010, KROHNE, Duisburg, Germany) while LVAD and RVAD outlet flow rates were recorded with clamp-on ultrasonic flow meters (TS410-10PXL, Transonic Systems, Ithaca, NY, USA). Circulatory and VAD pressures were recorded using silicone-based transducers (PX181B-015C5V, Omega Engineering, Stamford, CT, USA). 


\section{$\underline{\text { Results }}$}

Results of the experiment were analysed to observe the changes in RVAD rotational speed, power consumption, outlet pressure measured directly after the pump and at the interface between the outflow cannula and pulmonary artery (mean pulmonary artery pressure - MPAP) with increasing pulmonary vascular resistance.

\section{Severe RV failure}

As expected, an increase in outflow cannula resistance or PVR required increased pump speed to maintain a flow rate of $5 \mathrm{~L} / \mathrm{min}$ (Figure 2a). At a PVR of 40 dyne.s.cm ${ }^{-5}$ (0.5 Wood unit), the RVAD rotational speeds required to maintain 5L/min flow with a $20 \mathrm{~cm}$ outflow conduit with $5 \mathrm{~mm}$ banding and unbanded 60 $\mathrm{cm}, 40 \mathrm{~cm}$ and $20 \mathrm{~cm}$ outflow conduits were 3120, 2760, 2380 and $2160 \mathrm{rpm}$ respectively. This increased to 3480, 3280, 2860 and $2700 \mathrm{rpm}$ when PVR was increased to 560 dyne.s.cm ${ }^{-5}$ (7 Wood unit), with the increase in rotational speed following a progressive, linear relationship with PVR. The corresponding power consumption and pump outlet pressures behaved similarly. Power consumption at a PVR of 40 dyne.s.cm ${ }^{-5}$ was 7, 5.4, 4.5 and 3.7 Watts for the banded, $60 \mathrm{~cm}, 40$ $\mathrm{cm}$ and $20 \mathrm{~cm}$ conduits respectively, and 9.2, 8.3, 6.5 and 5.6 Watts at 560 dyne.s.cm ${ }^{-5}$ (Figure 2b).

RVAD outlet pressure generated at the lowest tested PVR setting were 78, 48, 45 and $30 \mathrm{mmHg}$, and 112, 86, 79 and $65 \mathrm{mmHg}$ in the highest (Figure 2c). This demonstrated that left-sided afterload was sufficiently simulated when the RVAD outflow was banded in the setting of severe RV failure. The unbanded conduits, however, resulted in reduced RVAD afterload of between $39 \%$ (60 cm conduit, 40 dyne.s.cm ${ }^{-5}$ ) and $77 \%$ (20 cm conduit, 560 dyne.s.cm ${ }^{-5}$ ) of that seen with the LVAD, depending on conduit length and PVR. However, the MPAP was similar in all outflow conduit conditions (Figure 2d). 
(a)

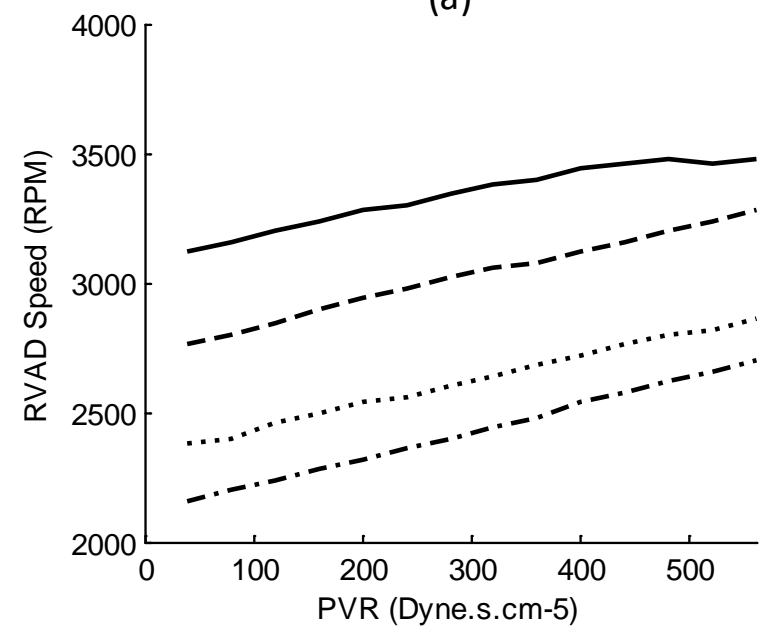

(c)

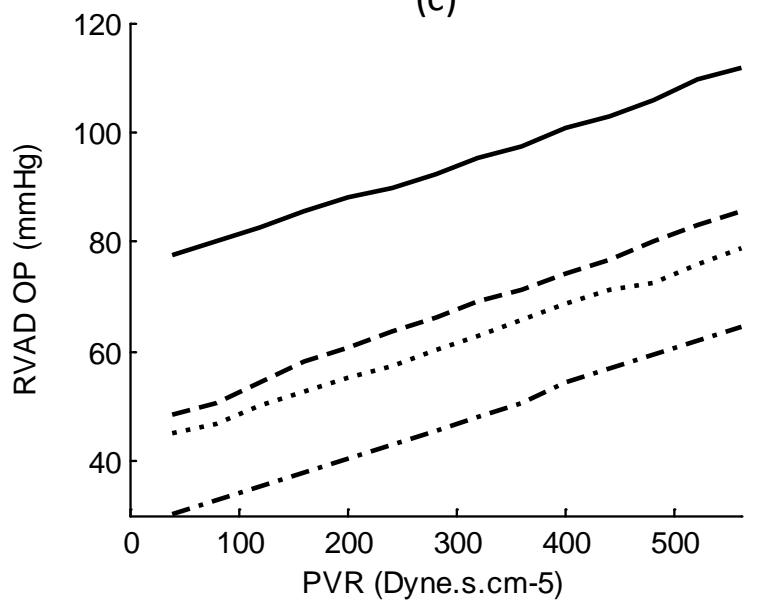

(b)

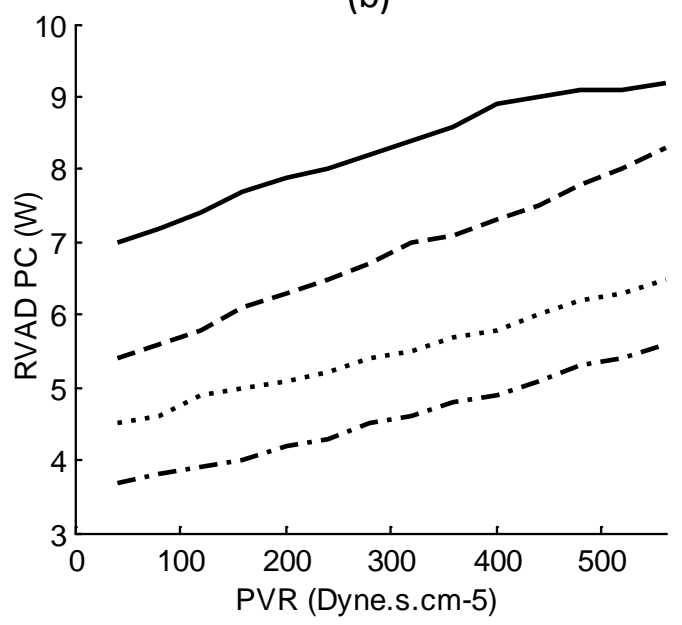

(d)

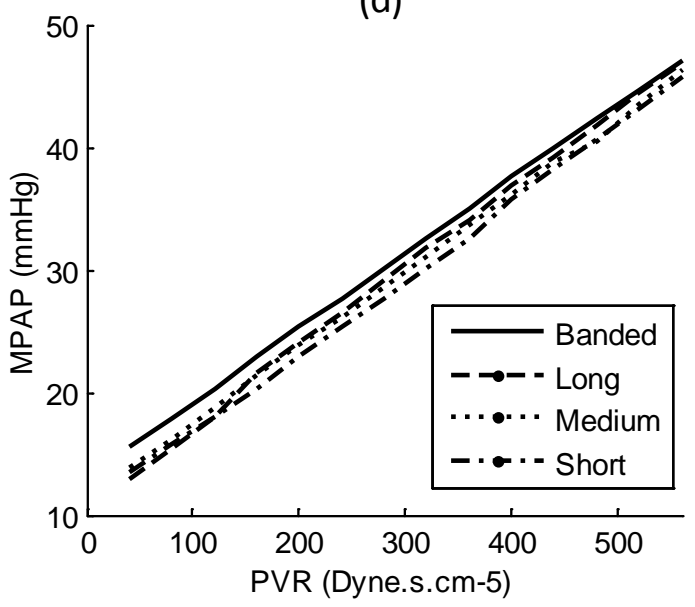

Figure 2 - Results for the severe biventricular heart failure condition with varying pulmonary vascular resistance (PVR) including (a) RVAD speed, (b) RVAD power consumption (PC), (c) RVAD outflow pressure (OP) and (d) mean pulmonary arterial pressure (MPAP). W - Watts. Recommended RVAD speed range of operation $2400-3200$ rpm.

\section{Mild RV failure}

Compared with severe RV failure, the same flow-rate and pressure was achieved with lower RVAD speed and power consumption in all outflow conduit conditions tested. With the exception of the unbanded conduits at a PVR of 40 dyne.s.cm-5, the RVAD was able to function above the manufacturer's recommended minimum rotational speed in the setting of mild RV failure (2400 rpm) (13) and produce $5 \mathrm{~L} / \mathrm{min}$ flow to match that of the LVAD (Figure 3a). This was similar to the findings in severe RV failure, where the shortest unbanded conduit required operation of the RVAD at a sub-optimal rotational speed. This effect was ameliorated with the application of $40 \mathrm{~cm}, 60 \mathrm{~cm}$ and banded conduits. 
Overall, in the tested outflow conduit conditions, the corresponding rotational speed, and subsequent power consumption and outlet pressure generated was, on average, lower in mild RV failure than that in severe RV failure. The mean difference in RVAD rotational speed between severe RV and mild RV failure was $1.9 \%$, mean difference in power consumption was $6.3 \%$ and mean difference in RVAD outlet pressure was 8.3\%. However, the relationship of these three variables with PVR in the setting of mild RV failure differed from that in severe RV failure. For instance, from 40 to 120 dyne.s. $\mathrm{cm}^{-5}$, the increase in mean RVAD rotational speed in the setting of mild RV failure was $22.7 \%$, but from 120 to 560 dyne.s.cm-5, the increase was only $4.0 \%$. This is opposed to the linear relationship between these variables and PVR in severe RV failure, and was more marked in the unbanded conduits. This can be attributed to the RV contributing to forward flow at low levels of PVR where the RV end diastolic volume is higher. Through the Starling response implemented in the MCL, this resulted in increased right ventricular contractility and subsequent ejection through the pulmonary valve. As PVR, and consequently MPAP, increased and ejection through the pulmonary valve decreased, greater changes in RVAD speed were required to overcome the reduced $\mathrm{RV}$ ejection and the increase in resistance. Once the RVAD was providing total circulatory support (PVR at 120 dyne.s.cm ${ }^{-5}$ ), smaller changes in pump speed were required to maintain pulmonary flow rate (Figure $3 b)$. 
(a)

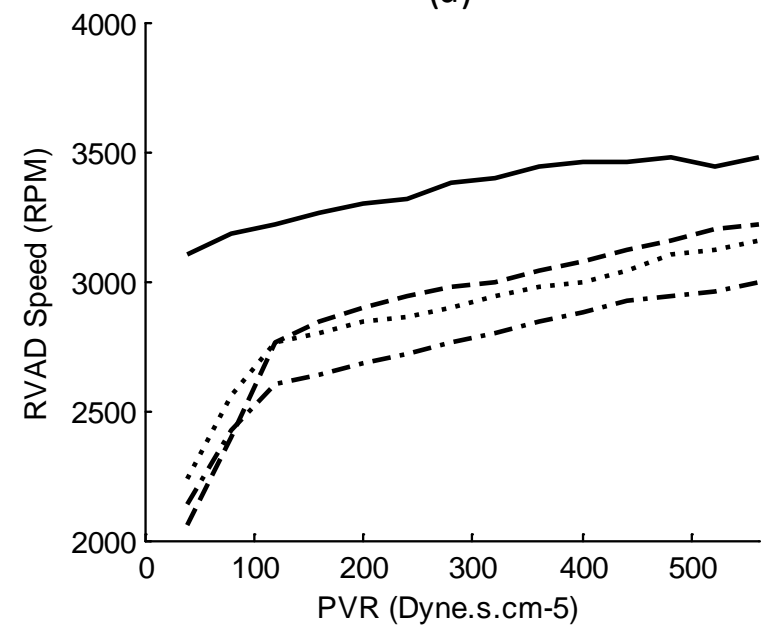

(c)

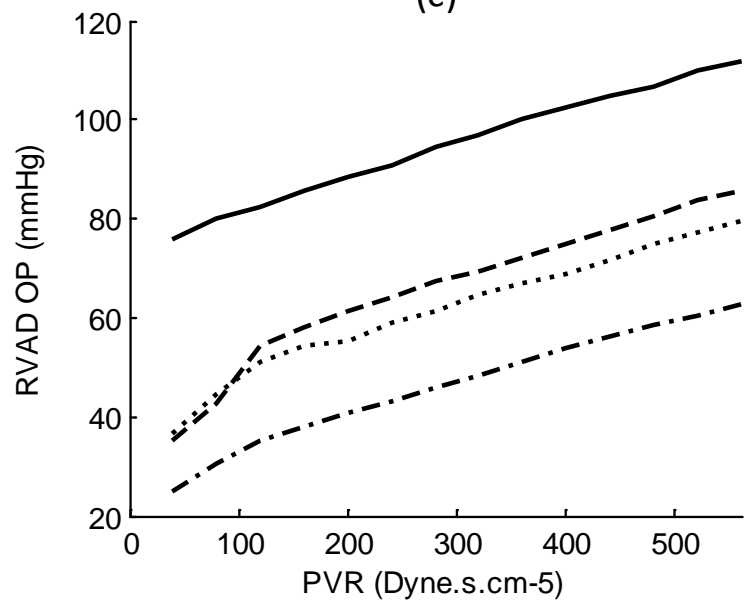

(b)

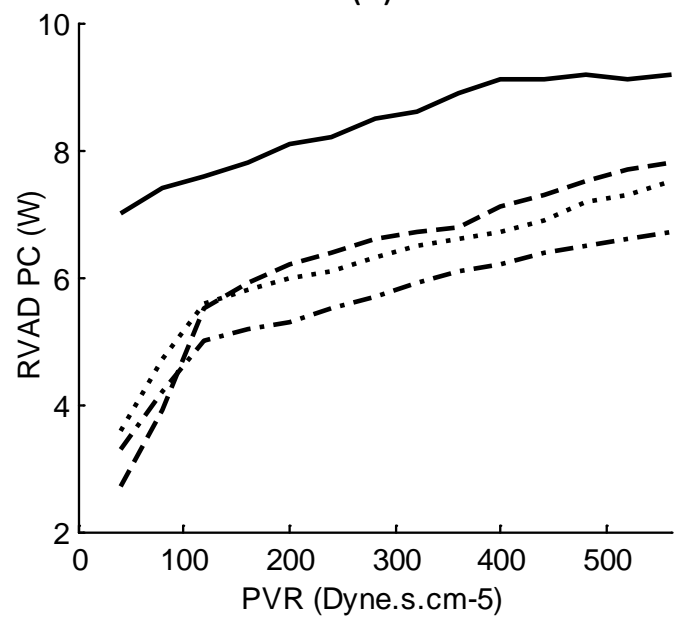

(d)

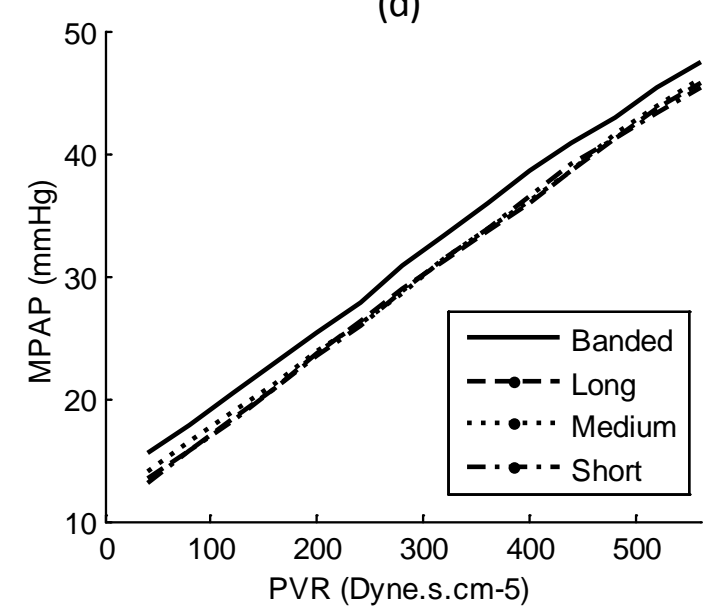

Figure 3 - Results for the mild biventricular heart failure condition with varying pulmonary vascular resistance (PVR) including (a) RVAD speed, (b) RVAD power consumption (PC), (c) RVAD outflow pressure (OP) and (d) mean pulmonary arterial pressure (MPAP). W - Watts. Recommended RVAD speed range of operation $2400-3200 \mathrm{rpm}$.

\section{Discussion}

Although various mechanical circulatory support devices have been reportedly used successfully for right ventricular support (14), there remains to be a commercially available RVAD-specific third generation device. While devices are still being developed $(15,16)$, adopting existing systems for RVAD use presents challenges in their set-up that must be overcome. This includes device placement (17), operational parameters and the outflow tract dimensions (18).

In the case of the HeartWare HVAD used in this study, the device is fitted with a hydrodynamically levitated impeller, relying on sufficient speed to create a fluid film and thus may become unstable at lower speeds (19). However, increasing 
RVAD rotational speed and flow rate raises pulmonary artery and capillary pressures (20). Multiple previous studies have assessed the necessary modifications to the HVAD for use and, similar with other devices (13), have concluded that outflow conduit restriction to simulate systemic afterload and speed alterations for fine-tuning can optimise pump function $(21,22)$.

While our clinical experience with banding has been successful, we note the anecdotal suggestions of surgeons attempting to vary conduit length to achieve the same result. This was justified using Poiseuille's law stating that resistance of a lumen is a function of radius and tube length. However, the required increase in length to achieve the same resistance did not seem realistic for implantation. To the knowledge of our group, there is little to no discussion of this in the literature previously. As such, we aimed to observe the performance of a third generation VAD with varying outflow conduit conditions and compare this with performance of a banded conduit.

In this experiment, we found that all tested outflow conduit conditions caused the pump to run outside of manufacturer's recommended rotational speed (2400 rpm to $3200 \mathrm{rpm}$ ) (13) at some degree of PVR. However, we found that the banded conduit had the narrowest range of PVR conditions where it would function within the recommendations (40 dyne.s. $\mathrm{cm}^{-5}$ to 120 dyne.s.cm ${ }^{-5}$ in severe RV failure, and 40 dyne.s. $\mathrm{cm}^{-5}$ to 80 dyne.s. $\mathrm{cm}^{-5}$ in mild RV failure) and achieve a normal haemodynamic state. Higher resistance conditions forced the pump to be operated faster than $3200 \mathrm{rpm}$. Conversely, the lowest resistance conduit also required pump speed outside of the prescribed parameters in the lowest PVR setting. The optimal outflow conduit configuration for maintaining pump function within the guidelines seemed to be the $40 \mathrm{~cm}$ unbanded conduit. This conduit had the widest range of PVR where it would facilitate pump operation within manufacturer's guidelines. In clinical terms, where PVR has a reference range of 155 to 255 dyne.s. $\mathrm{cm}^{-5}$ (23), a conduit of $40 \mathrm{~cm}$ should always function within manufacturer's guidelines based on our in-vitro results. This is 
important, as low-flow through devices have been reported as contributory factors to intra-pump thrombosis (24), while higher than recommended speeds result in excessive power consumption. We note that a variation in banding diameter may achieve a similar outcome. However, clinical practice, banding is often achieved by visual estimation rather than millimetre-perfect measurements. Variation of diameter is thus not a reliable and reproducible change like length variation. Furthermore, power consumption benefits appear to favour length variation. These results can be used as a guide for clinicians to base the intra-operative decision of RVAD outflow conduit set-up, as well as pump speed adjustments throughout the supported duration depending on changes in the patient's right ventricular function and afterload.

While the length of the outflow conduit impacted on RVAD afterload, it should be noted that the longest length tested $(60 \mathrm{~cm})$ only produced half the outlet pressure of a $5 \mathrm{~mm}$ diameter band. However, the practicality is that such a long outflow conduit would never be used clinically as it would be susceptible to kinking and compression from adjacent viscera. Testing at this length in an invitro setting is useful, though, to demonstrate the effects of varying graft length. Clinically, the shorter lengths can be achieved by laying the conduit down along the diaphragm and then up over the RV to reach the pulmonary trunk.

With the repetition of the test with mild RV failure, this study also took into account potential right-sided myocardial recovery and pulmonary vascular bed remodeling. We found that, even under improved right heart conditions, the RVAD did not need to drop below the recommended functional parameters to continue to produce an optimal haemodynamic state by simply extending the graft length. With complete myocardial recovery, there is evidence in the literature that the HVAD can be safely turned off without explantation (25). 
Stevens et al. evaluated the use of biventricular VentrAssist devices (Ventracor Ltd, Sydney NSW, Australia) with the right-sided device operated with reduced speeds or normal LVAD speeds with and without outflow banding (19). While this group's in-vitro and in-vivo findings were congruent with our own, the specific changes are not compatible simply because of differing pump design. In their experiment, the right-sided VentrAssist device was required to run at speeds below manufacturer's specification. This was not observed in our experiment. Furthermore, their animal study demonstrated increased RVAD afterload compared to the same degree of RV failure and PVR. Post-mortum examination revealed longer graft length and a thin, uniform layer of thrombus inside the graft as the likely causes, thus effectively banding the RVAD outflow conduit. If this were also true with the HeartWare device, we hypothesise that it would function well within manufacturer's guidelines in-vivo under all of our tested conditions.

As an in-vitro study, we were limited by multiple factors that were impossible to simulate. The complex auto-regulatory systems of a biological circulation are only partially replicated. Also, while our system simulates the viscosity of blood in a healthy human, this can vary significantly with heart failure. Furthermore, the coagulation properties of blood were not simulated, which, as aforementioned, can have significant impact on outflow conduit conditions in an in-vivo setting.

As expected, the capacity to run the pumps at lower speeds also brings with it power consumption benefits. We found that the difference in power consumption was between $9.8 \%$ and $48 \%$ lower by varying cannula length rather than banding while operating the pump within recommended parameters. However, the increased power consumption with RVAD outflow banding could be reduced by decreasing the RVAD outflow restriction and thus pump speeds. 
We defined the minimum pump speeds required to maintain $5 \mathrm{~L} / \mathrm{min}$ flow with a variety of outflow conduits and made the same, incremental changes to the PVR in all conduit conditions. As a result, a consistent mPAP trend was maintained between study conditions. However, based on the literature, we postulate that the pump speed would be higher in an in-vivo setting. Therefore, in-vivo evaluation of outflow banding against graft length with varying PVR is necessary to confirm our results. Furthermore, this will allow us to evaluate other impacts of banding versus graft length variation, such as blood damage.

\section{Conclusion}

In this study, using a sophisticated simulation of the human circulatory system, the HeartWare HVAD in an RVAD configuration was demonstrated to maintain physiological pulmonary flow rates with and without outflow cannula restriction in both severe and mild RV systolic dysfunction with varying levels of PVR. Increased length of the outflow conduit was found to produce significantly increased afterload to the device, and a graft length of $40 \mathrm{~cm}$ was found to provide optimal conditions for pump function. While results of this in-vitro study should be interpreted with caution, our results suggest that the utility of outflow conduit modification in the HeartWare HVAD may not be as great as previously believed. Further testing of this hypothesis in animal models is thus warranted to confirm these findings, and for the ongoing development of right ventricular mechanical support.

\section{CONFLICTS STATEMENT}

We, the authors, declare no conflicts of interests in writing and submitting this abstract.

\section{$\underline{\text { References }}$}

1. Rose EA, Gelijns AC, Moskowitz AJ, Heitjan DF, Stevenson LW, Dembitsky $\mathrm{W}$, et al. Long-term use of a left ventricular assist device for end-stage heart 
failure. The New England journal of medicine. 2001 Nov 15;345(20):1435-43. PubMed PMID: 11794191.

2. Mangi AA. Right ventricular dysfunction in patients undergoing left ventricular assist device implantation: predictors, management, and device utilization. Cardiology clinics. 2011 Nov;29(4):629-37. PubMed PMID: 22062214. Epub 2011/11/09. eng.

3. Fitzpatrick JR, 3rd, Frederick JR, Hiesinger W, Hsu VM, McCormick RC, Kozin ED, et al. Early planned institution of biventricular mechanical circulatory support results in improved outcomes compared with delayed conversion of a left ventricular assist device to a biventricular assist device. The Journal of thoracic and cardiovascular surgery. 2009 Apr;137(4):971-7. PubMed PMID: 19327526. Pubmed Central PMCID: 3232461.

4. Tsukui H, Teuteberg JJ, Murali S, McNamara DM, Buchanan JR, Winowich $\mathrm{S}$, et al. Biventricular assist device utilization for patients with morbid congestive heart failure: a justifiable strategy. Circulation. 2005 Aug 30;112(9 Suppl):I6572. PubMed PMID: 16159867.

5. Timms D, Gude E, Gaddum N, Lim E, Greatrex N, Wong K, et al. Assessment of right pump outflow banding and speed changes on pulmonary hemodynamics during biventricular support with two rotary left ventricular assist devices. Artificial organs. 2011 Aug;35(8):807-13. PubMed PMID: 21726243.

6. Gregory SD, Timms D, Gaddum N, Mason DG, Fraser JF. Biventricular assist devices: a technical review. Annals of biomedical engineering. 2011 Sep;39(9):2313-28. PubMed PMID: 21739329.

7. Aissaoui N, Borgermann J, Gummert J, Morshuis M. HeartWare continuous-flow ventricular assist device thrombosis: the Bad Oeynhausen experience. The Journal of thoracic and cardiovascular surgery. 2012 Apr;143(4):e37-9. PubMed PMID: 22245238.

8. Timms DL, Gregory SD, Greatrex NA, Pearcy MJ, Fraser JF, Steinseifer U. A compact mock circulation loop for the in vitro testing of cardiovascular devices. Artificial organs. 2011 Apr;35(4):384-91. PubMed PMID: 20883450.

9. Gregory SG, N.; Timms, D.; Gaddum, N.; Pearcy, M.; Fraser, J. Simulation and enhancement of a cardiovascular device test rigt. Journal of Simulation. 2010;4:34-41.

10. Gregory SD, Stevens M, Timms D, Pearcy M. Replication of the FrankStarling response in a mock circulation loop. Conference proceedings : Annual International Conference of the IEEE Engineering in Medicine and Biology Society IEEE Engineering in Medicine and Biology Society Conference. 2011;2011:6825-8. PubMed PMID: 22255906.

11. Klabunde RE. Cardiovascular physiology concepts. 2nd ed. Philadelphia, PA: Lippincott Williams \& Wilkins/Wolters Kluwer; 2012. xi, 243 p. p.

12. Segur JBO, H.E. Viscosity of Glycerol and Its Aqueous Solutions. Industrial and Engineering Chemistry. 1951 September 1, 1951;43(9):2117-20.

13. HeartWare Ventricular Assist System Instructions for Use. In: HeartWare I, editor. Miami Lakes, Florida 33014, United States of America: HeartWare, Inc.; 2012.

14. Hsu PL, Parker J, Egger C, Autschbach R, Schmitz-Rode T, Steinseifer U. Mechanical circulatory support for right heart failure: current technology and future outlook. Artificial organs. 2012 Apr;36(4):332-47. PubMed PMID:

22150419. 
15. Ootaki Y, Saeed D, Ootaki C, Akiyama M, Fumoto H, Horai T, et al. Development of the DexAide right ventricular assist device inflow cannula. ASAIO J. 2008 Jan-Feb;54(1):31-6. PubMed PMID: 18204313.

16. Fukamachi K, Ootaki Y, Horvath DJ, Massiello AL, Kamohara K, Akiyama M, et al. Progress in the development of the DexAide right ventricular assist device. ASAIO J. 2006 Nov-Dec;52(6):630-3. PubMed PMID: 17117051.

17. Schlensak C, Schibilsky D, Siepe M, Brehm K, Klemm R, von Wattenwyl R, et al. Biventricular cannulation is superior regarding hemodynamics and organ recovery in patients on biventricular assist device support. The Journal of heart and lung transplantation : the official publication of the International Society for Heart Transplantation. 2011 Sep;30(9):1011-7. PubMed PMID: 21489816.

18. Hetzer R, Krabatsch T, Stepanenko A, Hennig E, Potapov EV. Long-term biventricular support with the heartware implantable continuous flow pump. The Journal of heart and lung transplantation : the official publication of the International Society for Heart Transplantation. 2010 Jul;29(7):822-4. PubMed PMID: 20417132.

19. Stevens MC, Gregory SD, Nestler F, Thomson B, Choudhary J, Garlick B, et al. In Vitro and In Vivo Characterization of Three Different Modes of Pump Operation When Using a Left Ventricular Assist Device as a Right Ventricular Assist Device. Artificial organs. 2014 Mar 24. PubMed PMID: 24660783.

20. Punnoose L, Burkhoff D, Rich S, Horn EM. Right ventricular assist device in end-stage pulmonary arterial hypertension: insights from a computational model of the cardiovascular system. Progress in cardiovascular diseases. 2012 Sep-Oct;55(2):234-43 e2. PubMed PMID: 23009919.

21. Krabatsch T, Hennig E, Stepanenko A, Schweiger M, Kukucka M, Huebler $\mathrm{M}$, et al. Evaluation of the HeartWare HVAD centrifugal pump for right ventricular assistance in an in vitro model. ASAIO J. 2011 May-Jun;57(3):183-7. PubMed PMID: 21336105.

22. Strueber M, Meyer AL, Malehsa D, Haverich A. Successful use of the HeartWare HVAD rotary blood pump for biventricular support. The Journal of thoracic and cardiovascular surgery. 2010 Oct;140(4):936-7. PubMed PMID: 20478575.

23. Price LC, Wort SJ, Finney SJ, Marino PS, Brett SJ. Pulmonary vascular and right ventricular dysfunction in adult critical care: current and emerging options for management: a systematic literature review. Crit Care. 2010;14(5):R169. PubMed PMID: 20858239. Pubmed Central PMCID: 3219266.

24. Decoene C, Fayad G, Al-Ruzzeh S, Modine T, Crepin F, Pol A, et al. Right ventricular assist device thrombosis during biventricular heart assistance. Perfusion. 2004 Nov;19(6):365-7. PubMed PMID: 15619970.

25. Potapov E, Schweiger M, Vierecke J, Dandel M, Stepanenko A, Kukucka M, et al. Discontinuation of HeartWare RVAD support without device removal in chronic BIVAD patients. ASAIO J. 2012 Jan-Feb;58(1):15-8. PubMed PMID: 22210646. 